\title{
A New Coherent Similarity Measure for Temporal Multichannel Scene Characterization
}

\author{
Esra Erten, Andreas Reigber, Senior Member, IEEE, Laurent Ferro-Famil, Member, IEEE, and \\ Olaf Hellwich, Senior Member, IEEE
}

\begin{abstract}
This paper proposes a new method for a measure of coherent similarity between temporal multichannel synthetic aperture radar (SAR) images and its implementation to change detection application. The method is based on mutual information (MI) from information theory. The MI measures the amount of information in common between coherent temporal multichannel SAR acquisitions. In order to develop an algorithm for all kinds of SAR images, such as interferometric SAR, polarimetric-interferometric SAR (PolInSAR), and partial PolInSAR, first, the joint density function of temporal multichannel images based on their second-order statistics has been derived. Then, the derived joint density function is used to calculate an analytical expression for the MI between temporal images, which is assumed to be maximal if the temporal images are identical. Although, in this paper, a new coherent similarity measure has analytically been derived for temporal polarimetric SAR images based on complex Wishart process in time, since the mathematical formulation is general, it can equally well be implemented into any kind of multivariate remote sensing data, such as multispectral optical and interferometric images after small continuation. This derived quantity has been implemented for change detection application whose aim is to characterize the temporal behavior of the acquisitions. A comparison between the proposed and the other well-known change detection methods by means of scene characterization is shown, describing the advantages due to the fact that the proposed change detector involves almost every facet of applied change detection.
\end{abstract}

Index Terms-Change detection algorithms, mutual information, parameter estimation, polarimetric synthetic aperture radar.

\section{INTRODUCTION}

$\mathbf{T}$ HE SPECIAL case of temporal scene characterization known as change detection mapping is one of the core applications in the field of remote sensing considering that natural objects are characterized by their spatial patterns and also by their temporal dynamics, i.e., changing backscattering properties due to their inherent properties. Even though many

Manuscript received September 19, 2010; revised January 19, 2011, May 25, 2011, and July 11, 2011; accepted October 23, 2011 Date of publication December 14, 2011; date of current version June 20, 2012.

E. Erten is with the Chair of Earth Observation and Remote Sensing, Institute of Environmental Engineering, Swiss Federal Institute of Technology (ETH) Zurich, 8093 Zurich, Switzerland (e-mail: erten@ifu.baug.ethz.ch).

A. Reigber is with the Microwaves and Radar Institute, German Aerospace Center (DLR), 82230 Wessling, Germany (e-mail: andreas.reigber@dlr.de).

L. Ferro-Famil is with the Institute of Electronics and Telecommunications of Rennes, University of Rennes 1, 35042 Rennes, France (e-mail: Laurent.ferro-famil@univ-rennes1.fr).

O. Hellwich is with the Department of Computer Vision and Remote Sensing, Technische Universität Berlin, 10587 Berlin, Germany (e-mail: hellwich@cs.tu-berlin.de).

Color versions of one or more of the figures in this paper are available online at http://ieeexplore.ieee.org.

Digital Object Identifier 10.1109/TGRS.2011.2174155 studies are based on multispectral remote sensing images [1], [2], the availability and the efficiency of optical data sets are often limited by illumination and weather conditions. Hence, for change detection applications, temporal multichannel synthetic aperture radar (SAR) images become more attractive since the backscatter properties of SAR are almost independent of weather conditions. Additionally, the utilization of temporal multichannel SAR data is favored in order to make use of their inherent properties for a better temporal analysis of surface scattering.

Although the interpretation of SAR data is more difficult than that of multispectral imagery, several studies have assessed the positive impact of temporal SAR imaging on change detection applications [3]-[6]. In particular, temporal multichannel change detection techniques are interesting considering their inherent properties which are independent of SAR imaging mode, such as the generalized variance ratio [7] and the maximum-likelihood ratio test (MLRT) [5], [8], [9]. In the case of comparing the variabilities of two multivariate populations, a natural measure is the ratio of their generalized variances: For temporal single-channel images, it is the ratio of their intensities, and for temporal multichannel images, it is the ratio of determinants of covariance matrices of polarimetric SAR (PolSAR) images. Although this ratio image is very robust to speckle noise, it is not sufficient to characterize different kinds of temporal changes. For small changes in temporal intensities, the technique suffers from a significant false-alarm rate. Such test statistics can, for example, be used to test the equality of mean power of temporal images. The MLRT has been used to test the equality of temporal polarimetric covariance matrices, which follow complex Wishart distributions. It is equivalent to testing the equality of two Gamma-distributed intensity images for temporal single-channel images. Because this technique takes into account much more higher statistics, it can deal with different types of change. This technique is very powerful in the presence of step changes, such as ship detection, vehicle detection in forest, and diseased plant monitoring. Like the generalized variance ratio, the MLRT describes also incoherent resemblance of temporal images. In both techniques, PolSAR data improve the detection performance compared to singlechannel information by exploiting the wave polarization, allowing for a more sophisticated physical interpretation of temporal SAR data sets.

As discussed in the previous paragraph, taking into account higher order statistics improves the characterization of temporal behavior of data sets, so it is more useful to consider the probability density function (pdf) of the temporal images' pixels. This 
is why various similarity measures for pdf have been used in change detection applications. One of the similarity measures from information theory for multivariate populations is the Kullback-Leibler (KL) divergence. KL divergence is a noncommutative measure of the difference between two probability distributions, and its interesting implementation to SAR images can be seen in recent publications, such as [10] and [11]. KL divergence has been used for contrast analysis of multichannel SAR images in [10] and for change detection application in [11] by noting that a multiscale approach will improve the detection performance. Even though previous works mainly concentrated on the KL divergence, here, instead of the KL divergence, another measure from information theory called mutual information (MI) will be used to determine temporal similarity between multichannel SAR images. The MI is a basic concept from information theory, quantifying the mutual dependence of the two random variables.

MI has been previously used in coregistration of medical images [12] and recently used in fusion of optical and SAR images [13], [14]. This paper expands the ideas first presented in [15] which uses the MI to detect regions of change in video surveillance. In this work, the MI scalar is the state variable used to describe the coherent similarity between temporal multichannel SAR images by making use of the second-order statistics of the acquisitions. This paper is organized as follows. Section II reviews the principles of temporal SAR vector acquisitions in detail and includes the derivation of the joint density function of temporal polarimetric covariance matrices. The theoretical concept of the new coherent similarity measure by means of the MI is presented in Section III, whereas the implementation of this measure for real data is described in Section IV. In this section, the other well-known change detection technique called the MLRT is also reviewed to underline the potential of the MI to characterize the scene change between temporal acquisitions. Eventually, Section V concludes this paper with some discussions and some directions for future works.

\section{Polarimetric-Interferometric Image Model}

\section{A. Acquisition Vector}

Let a temporal acquisition vector $\vec{k}=\left[\begin{array}{ll}\vec{k}_{1} & \vec{k}_{2}\end{array}\right]^{\mathrm{T}}$ be a complex vector distributed as a multicomponent circular Gaussian $\mathcal{N}^{C}(0, \Sigma)$ that consists of two target vectors $\vec{k}_{1} \sim \mathcal{N}^{C}\left(0, \Sigma_{11}\right)$ and $\vec{k}_{2} \sim \mathcal{N}^{C}\left(0, \Sigma_{22}\right)$ obtained from temporal multichannel SAR images at times $t_{1}$ and $t_{2}$, respectively. These two observations can be correlated or uncorrelated processes over time depending on the monitored objects. Here, the number of elements in one of the target vectors $\vec{k}_{i}$ at time $t_{i}$ is represented by $m$, and hence, the temporal target vector $\vec{k}$ has the dimension of $q=2 \times m$. For example, $q=2$ corresponds to interferometric SAR (InSAR) images, whereas $q=6$ corresponds to polarimetric-interferometric SAR (PolInSAR) images. It can be remembered that, with single-channel data $(m=1)$, only one copolarized channel $\vec{k}_{i}=\vec{k}_{h h}$ or $\vec{k}_{i}=\vec{k}_{v v}$ is recorded, and the phase carries no useful information for distributed targets. When multichannel (polarimetric) data are available, e.g., $\vec{k}_{i}=\left[\begin{array}{lll}k_{h h} & k_{h v} & k_{h v}\end{array}\right]$, phase differences between channels provide information about dielectric and geometric properties of the scattering medium [16].

The true covariance matrix $\Sigma$, which contains sufficient statistics to characterize the acquisition vector $\vec{k}$, is not known and is estimated using a maximum-likelihood method by $n$-sample ( $n$-look) spatial coherent averaging: $A=$ $(1 / n) \sum_{j=1}^{n} \vec{k}_{j} \vec{k}_{j}^{\dagger}$. The true covariance matrix $\Sigma$, as well as its $n$-sample estimate $A$, can be portioned as

$$
\Sigma=\left[\begin{array}{ll}
\Sigma_{11} & \Sigma_{12} \\
\Sigma_{21} & \Sigma_{22}
\end{array}\right] \quad A=\left[\begin{array}{ll}
A_{11} & A_{12} \\
A_{21} & A_{22}
\end{array}\right]
$$

which summarize all the information (joint and marginal) from temporal multichannel SAR acquisitions. $A_{12}=A_{21}^{\dagger}$ is an $m \times m$-dimensional cross-correlation matrix between the acquisition vectors $\vec{k}_{1}$ and $\vec{k}_{2}$ which characterizes the interferometric and polarimetric information. The matrices $A_{11}$ and $A_{22}$ are the standard $n$-look and $m \times m$-dimensional polarimetric covariance matrices of separate temporal images. The pdf of the $n$-sample covariance matrix $A$, known as a complex Wishart distribution with $n$ degrees of freedom, is

$$
p(A) \sim \mathcal{W}^{C}(n, \Sigma)=\frac{n^{m n}|A|^{n-m}}{|\Sigma|^{n} \tilde{\Gamma}_{m}(n)} \operatorname{etr}\left(-n \Sigma^{-1} A\right)
$$

provided that $n \geq m$ [17]. Here, etr and $|\cdot|$ denote the exponential trace and the determinant of the positive definite covariance matrix, respectively, and the normalization constant $\tilde{\Gamma}_{m}(n)$ is the multivariate Gamma function

$$
\tilde{\Gamma}_{m}(n)=\pi^{m(m-1) / 2} \prod_{i=1}^{m} \Gamma(n-i+1) .
$$

Substituting $m=1$ into (2), the well-known single-channel multilook intensity distribution, named the Gamma distribution, is obtained where $n$-look polarimetric covariance matrix $A_{i i}$ reduces to the $n$-look single-channel intensity image $a_{i}$. Hence, all the properties of the Gamma distribution are simple consequences of those of the complex Wishart distribution.

\section{B. Joint Density Function of Temporal Polarimetric Covariance Matrices: $p\left(A_{11}, A_{22}\right)$}

The joint statistics of complex Wishart matrices in time gives the theoretical basis required to study the time-varying behavior of SAR images, owing to the MI. The properties of the Hermitian matrix $A$ in (1) permit deriving the joint pdf of the temporal covariance matrices $A_{11}$ and $A_{22}$ as in the following.

The conditional pdf of $A_{11}$ given $A_{22}$ follows the complex Wishart distribution with $n-m$ degrees of freedom [17]

$$
\begin{aligned}
A_{11 \mid 22} & =A_{11}-A_{12} A_{22}^{-1} A_{21} \\
p\left(A_{11} \mid A_{22}\right) & =\mathcal{W}^{C}\left(n-m, \Sigma_{11 \mid 22}\right)
\end{aligned}
$$

where $\Sigma_{11 \mid 22}=\Sigma_{11}-\Sigma_{12} \Sigma_{22}^{-1} \Sigma_{21}$. Moreover, as the sample covariance matrix of $A_{22}$ follows a complex Wishart density function with $n$ degrees of freedom $\mathcal{W}^{C}\left(n, \Sigma_{22}\right)$, the 
conditional probability density of $A_{12}$ given $A_{22}$ is a complex normal function [18]

$$
p\left(A_{12} \mid A_{22}\right) \sim \mathcal{N}^{\mathcal{C}}\left(\Sigma_{12} \Sigma_{22}^{-1} A_{22}, \Sigma_{11 \mid 22} \otimes A_{22}\right)
$$

where $\otimes$ denotes the Kronecker product of the matrices. Due to the fact that any linear transformation of a normal vector has a normal distribution, multiplying (4) with $A_{22}^{-1 / 2}$ yields

$$
p\left(A_{12} A_{22}^{-1 / 2} \mid A_{22}\right)=\mathcal{N}^{\mathcal{C}}\left(\Sigma_{12} \Sigma_{22}^{-1} A_{22}^{1 / 2}, \Sigma_{11 \mid 22} \otimes I_{m}\right) .
$$

Here, $I_{m}$ is the $m \times m$ identity matrix. Since $A_{12}=A_{21}^{\dagger}$, $A_{12} A_{22}^{-1} A_{21}$ can be formulated as $A_{12} A_{22}^{-1 / 2}\left(A_{12} A_{22}^{-1 / 2}\right)^{\dagger}$. Then, $p\left(A_{12} A_{22}^{-1} A_{21} \mid A_{22}\right)$ follows a complex noncentral Wishart distribution with $m$ degrees of freedom and noncentrality parameter $\Sigma_{12} \Sigma_{22}^{-1} A_{22} \Sigma_{22}^{-1} \Sigma_{21}$. Hereafter, the shorthand $W \sim \mathcal{W}^{\mathcal{C}}(b, \Phi, \Theta)$ is used to express that $W=S S^{\dagger}$ follows the noncentral complex Wishart distribution according to the pdf $[19$, Def. II]

$$
p(W)=\operatorname{etr}(-\Theta)_{0} \tilde{F}_{1}\left(b, \Theta \Phi^{-1} W\right) \underbrace{\frac{\operatorname{etr}\left(-\Phi^{-1} W\right)|W|^{b-c}}{\tilde{\Gamma}_{c}(b)|\Phi|^{b}}}_{\mathcal{W}^{\mathcal{C}}(b, \Upsilon)}
$$

where the $b \times c$ matrix $S$ is $\mathcal{N}^{c}\left(\Upsilon, \Phi \otimes I_{b}\right)$ with $c \leq b$ and $\Theta=$ $\Phi^{-1} \Upsilon \Upsilon^{\dagger}$ is the matrix of noncentrality parameters. With the help of this definition, $p\left(A_{12} A_{22}^{-1} A_{21} \mid A_{22}\right)$ can be expressed as

$$
\begin{aligned}
p\left(A_{12}\right. & \left.A_{22}^{-1} A_{21} \mid A_{22}\right) \\
& =\mathcal{W}^{\mathcal{C}}\left(m, \Sigma_{11 \mid 22}, \Sigma_{12} \Sigma_{22}^{-1} A_{22} \Sigma_{22}^{-1} \Sigma_{21} \Sigma_{11 \mid 22}^{-1}\right) .
\end{aligned}
$$

Writing $A_{12} A_{22}^{-1} A_{21}=D$, one then obtains the density function of $p\left(A_{11 \mid 22}, A_{22}, D \mid A_{22}\right)$ as

$$
p\left(A_{11 \mid 22}, A_{22}, D \mid A_{22}\right)=p\left(A_{11 \mid 22}\right) p\left(A_{22}\right) p\left(D \mid A_{22}\right) .
$$

Thus, from (2), (3), and (7) follows

$$
\begin{aligned}
p\left(A_{11 \mid 22}, A_{22}, \mathcal{D} \mid A_{22}\right) & \frac{n^{m n}\left|A_{22}\right|^{n-m} \exp \left(-n \operatorname{tr} \Sigma_{22}^{-1} A_{22}\right)}{\left|\Sigma_{22}\right|^{n} \tilde{\Gamma}_{m}(n)} \\
& \times \frac{n^{m(n-m)}\left|A_{11 \mid 22}\right|^{n-2 m} \exp \left(-n \operatorname{tr} \Sigma_{11 \mid 22}^{-1} A_{11 \mid 22}\right)}{\left|\Sigma_{11 \mid 22}\right|^{n-m} \tilde{\Gamma}_{m}(n-m)} \\
& \times \frac{\exp \left(-n \operatorname{tr} \Sigma_{11 \mid 22}^{-1} D-n \operatorname{tr} \Sigma_{11 \mid 22}^{-1} \Sigma_{12} \Sigma_{22}^{-1} A_{22} \Sigma_{22}^{-1} \Sigma_{21}\right)}{\left|\Sigma_{11 \mid 22}\right|^{m} \tilde{\Gamma}_{m}(m)} \\
& \times{ }_{0} \tilde{F}_{1}(\underbrace{m, n^{2} \Sigma_{11 \mid 22}^{-1} \Sigma_{12} \Sigma_{22}^{-1} A_{22} \Sigma_{22}^{-1} \Sigma_{21} \Sigma_{11 \mid 22}^{-1} D}_{M}) .
\end{aligned}
$$

Here, ${ }_{0} \tilde{F}_{1}(s, M)$ is the complex hypergeometric function of matrix $M$ and closely related to Bessel functions. This function can be calculated with the help of the positive eigenvalues of the Hermitian matrix $M$ by [20]

$$
\begin{aligned}
{ }_{0} \tilde{F}_{1}(s, t)=(-1)^{m(m-1) / 2} \prod_{k=1}^{m}(s-k) ! \\
\times \frac{\left|t_{j}^{(m-s+i-1) / 2} I_{s-m+i-1}\left(2 \sqrt{t_{j}}\right)\right|_{i, j=1}^{m}}{\prod_{i<j}^{m}\left(t_{i}-t_{j}\right)}
\end{aligned}
$$

where $I_{\alpha}(x)$ is a hypergeometric Bessel function of the first kind and $t_{m}<t_{m-1}<\cdots<t_{1}$ are the positive eigenvalues of the $m \times m$ Hermitian matrix $M$. Substituting $A_{11}=A_{11 \mid 22}+D$ and $R^{2}=A_{11}^{-1 / 2} A_{12} A_{22}^{-1} A_{21} A_{11}^{-1 / 2}$ into (9) and applying the rule of the change of variables [17, Th. 2.1.5]

$$
\begin{aligned}
R^{2} & =A_{11}^{-1 / 2} D A_{11}^{-1 / 2} \\
d R^{2} & =\left|A_{11}\right|^{m} d D,
\end{aligned}
$$

$p\left(A_{11}, A_{22}, R^{2}\right)$ can be written as in (11), shown at the bottom of the page, with $P^{2}=\Sigma_{11}^{-1 / 2} \Sigma_{12} \Sigma_{22}^{-1} \Sigma_{21} \Sigma_{11}^{-1 / 2}$. It is clear that (11) is valid under the conditions $A_{11}, A_{22}>0$ and $0<$ $R^{2}<I_{m}$, which means that $A_{11}, A_{22}, R^{2}$, and $I_{m}-R^{2}$ are the positive definite matrices. ${ }^{1}$ Integrating $R^{2}$ over (11) with the help of [22, eq. A.3.1], the joint distribution of temporal multichannel SAR images is given by

$$
p\left(A_{11}, A_{22}\right)=\int_{0<R^{2}<I_{m}} p\left(A_{11}, A_{22}, R^{2}\right) d R^{2}
$$

following (13). In single-channel SAR imaging mode, i.e., $m=$ 1, $p\left(A_{11}, A_{22}\right)$ may be rewritten in a simpler closed form, as a bivariate Gamma distribution $p\left(a_{1}, a_{2}\right)$ with common shape parameter $n>0$, scale parameters $\sigma_{1}>0$ and $\sigma_{2}>0$, and correlation coefficient $\rho^{2}=\left(\sigma_{12}^{2} / \sigma_{1} \sigma_{2}\right)$ [23]. By setting $m=1$ in (12) and substituting (10) into (12), the bivariate Gamma distribution (13), shown at the bottom of the next page, is obtained. Such an expression has already been used to detect the changes and to register single-channel temporal SAR images, as stated in [24].

Having introduced, the joint density of polarimetric covariance matrices $p\left(A_{11}, A_{22}\right)$ can be plotted in a simple case and compared to that with Monte Carlo simulations. Fig. 1 shows the comparison between the theoretical bivariate distribution

\footnotetext{
${ }^{1}$ The joint distribution of two real Wishart-distributed samples can be found in [21]. The joint distribution of two complex Wishart-distributed samples with the condition of $\Sigma_{11}=\Sigma_{22}$ is presented in [20]. However, the joint distribution function of the second-order statistics of two PolSAR images is not clearly defined since, generally, $\Sigma_{11} \neq \Sigma_{22}$.
}

$p\left(A_{11}, A_{22}, R^{2}\right)=\frac{\operatorname{etr}\left(-n \Sigma_{11 \mid 22}^{-1} A_{11}\right) \operatorname{etr}\left(-n \Sigma_{22 \mid 11}^{-1} A_{22}\right){ }_{0} \tilde{F}_{1}\left(m, n^{2} A_{11}^{1 / 2} \Sigma_{11 \mid 22}^{-1} \Sigma_{12} \Sigma_{22}^{-1} A_{22} \Sigma_{22}^{-1} \Sigma_{21} \Sigma_{11 \mid 22}^{-1} A_{11}^{1 / 2} R^{2}\right)}{\left|\Sigma_{22}\right|^{n} \tilde{\Gamma}_{m}(n) \tilde{\Gamma}_{m}(n-m)\left|\Sigma_{11}\right|^{n}\left|I_{m}-P^{2}\right|^{n} \tilde{\Gamma}_{m}(m)\left|I-R^{2}\right|^{q-n}\left|A_{11}\right|^{m-n} n^{-2 m n}\left|A_{22}\right|^{m-n}}$ 


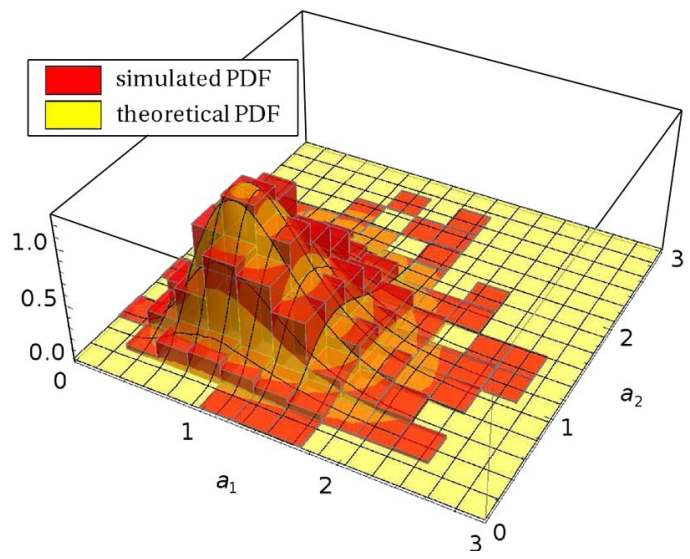

Fig. 1. Comparison between the theoretical bivariate distribution (12) with the simplest SAR imaging mode $(m=1)$ and the 2-D bivariate histogram from simulated data in the case of $n=9$. For simulation, the amplitudes and their correlation are given by $\sigma_{1}=\sigma_{2}=1$ and $\rho=0.1$, respectively. When $n \rightarrow$ $\infty, a_{1}=\sigma_{1}=1$, and $a_{2}=\sigma_{2}=1$.

derived in (12) and a simulated 2-D bivariate histogram. The Kolmogorov-Smirnov test, established as well [25], indicates an excellent fit between $p\left(a_{1}, a_{2}\right)$ and the simulated data, with a $p$ value of 0.92 . This fit may also be observed by a visual comparison of the pdfs shown in Fig. 1. Additionally, histograms obtained using a $3 \times 3$ estimation window over different agricultural fields in real images (Experimental-SAR (E-SAR) L-band) are compared with the theoretical density function (12) where the number of samples of eight has been found to provide the best fit to reconstructed histograms.

\section{New Coherent Similarity Measure BY MEANS OF MI}

In probability and information theory, the MI is a commutative measure of the difference between the joint probability distribution $p_{X, Y}(x, y)$ and the marginal probability distributions $p_{X}(x)$ and $p_{Y}(y)$ of the random variables $X$ and $Y$, respectively. The MI between $X$ and $Y$ is given by

$$
\begin{aligned}
\mathcal{D}_{\mathrm{MI}}(X ; Y) & =\int_{Y} \int_{X} \log \left(\frac{p_{X, Y}(x, y)}{p_{Y}(y) p_{X}(x)}\right) p_{X, Y}(x, y) d x d y \\
& =\mathcal{H}(X)-\mathcal{H}(X \mid Y) \\
& =\mathcal{H}(Y)-\mathcal{H}(Y \mid X)
\end{aligned}
$$

where $\mathcal{H}(X \mid Y)$ and $\mathcal{H}(Y \mid X)$ are the conditional entropies and $\mathcal{H}(X)$ and $\mathcal{H}(Y)$ are the marginal entropies of the random vari- ables $X$ and $Y$ [26]. The MI quantifies the dependence between the random variables $X$ and $Y$. Since entropies are regarded as a measure of uncertainty, $\mathcal{D}_{\mathrm{MI}}(X ; Y)$ simply measures how much knowing one of these variables reduces the uncertainty about the other.

Clearly, $\mathcal{H}(X) \geq \mathcal{H}(X \mid Y)$, and $\mathcal{H}(Y) \geq \mathcal{H}(Y \mid X)$ : Hence, $\mathcal{D}_{\mathrm{MI}}(X ; Y) \geq 0$. Additionally, the $\mathrm{MI}$ is a symmetric distance measurement: $\mathcal{D}_{\mathrm{MI}}(X ; Y)=\mathcal{D}_{\mathrm{MI}}(Y ; X)$. From (14), it follows that $\mathcal{D}_{\mathrm{MI}}(X ; Y)$ can be written as an expected value

$$
\mathcal{D}_{\mathrm{MI}}(X ; Y)=E\left\{\log \frac{p_{X, Y}(x, y)}{p_{Y}(y) p_{X}(x)}\right\}
$$

which takes its minimum in the case of $p_{X, Y}(x, y)=p_{Y}(y) \times$ $p_{X}(x)$ and its higher values if the joint density is sparse with localized peaks, implying that the two vectors are highly correlated and knowledge of one of them reduces the entropy with respect to the other.

The MI can be extended to the case where several parameters of the random variables $X$ and $Y$ are known. Moreover, it can also be implemented in the case of sequences of random vector variables, i.e., multichannel SAR acquisition vectors. In this case, the MI between two temporal acquisition random vectors is an appropriate tool to describe the temporal and spatial variations of the acquired signals characterized by their own local pdf.

Using the MI (14) as a measure of coherent similarity requires knowledge of the joint distribution function of the second-order statistics of temporal target vectors. Then, taking the derived joint density function, a new coherent similarity measurement may be formulated as

$$
\mathcal{D}_{\mathrm{MI}}^{n}\left(A_{11} ; A_{22}\right)=\int \log \left(\frac{p\left(A_{11}, A_{22}\right)}{p\left(A_{11}\right) p\left(A_{22}\right)}\right) p\left(A_{11}, A_{22}\right) d \vec{A}
$$

where $A$ is the vector including $2 m^{2}$ elements obtained by stacking the columns of $A_{11}$ and $A_{22}$ consecutively. Here, $n$ indicates the number of samples used in the estimation of the covariance matrices.

The analytical derivation of the MI can then be followed as in (16), shown at the bottom of the next page, by substituting the joint density (12) and the marginal densities (2) into (15) and using the equalities $\Sigma_{11 \mid 22}=\Sigma_{11}\left(I_{m}-P^{2}\right)$, $\Sigma_{22 \mid 11}=\Sigma_{22}\left(I_{m}-P^{2}\right), \quad$ and $\quad C=n^{2} A_{11}^{1 / 2} \Sigma_{11 \mid 22}^{-1} \Sigma_{12} \Sigma_{22}^{-1}$ $A_{22} \Sigma_{22}^{-1} \Sigma_{21} \Sigma_{11 \mid 22}^{-1} A_{11}^{1 / 2}$. Since the expectation of the sample covariance matrix $A_{i i}$ equals its true covariance matrix $\Sigma_{i i}$, i.e.,

$$
\begin{aligned}
p\left(A_{11}, A_{22}\right) & =\frac{\operatorname{etr}\left(-n \frac{\Sigma_{22}^{-1} A_{22}+\Sigma_{11}^{-1} A_{11}}{I-P^{2}}\right)\left|A_{11} A_{22}\right|^{n-m}{ }_{0} \tilde{F}_{1}\left(n, n^{2} A_{11}^{1 / 2} \Sigma_{11 \mid 22}^{-1} \Sigma_{12} \Sigma_{22}^{-1} A_{22} \Sigma_{22}^{-1} \Sigma_{21} \Sigma_{11 \mid 22}^{-1} A_{11}^{1 / 2}\right)}{n^{-2 m n}\left|\Sigma_{11} \Sigma_{22}\right|^{n}\left|I-P^{2}\right|^{n} \tilde{\Gamma}_{m}(n)^{2}} \\
p\left(a_{1}, a_{2}\right) & =\frac{n^{n+1} a_{1}^{\frac{n-1}{2}} a_{2}^{\frac{n-1}{2}} \rho^{\frac{1-n}{2}}}{\Gamma(n) \sigma_{1}^{\frac{n+1}{2}} \sigma_{2}^{\frac{n+1}{2}}(1-\rho)} I_{n-1}\left(\frac{2 n \sqrt{\rho}}{1-\rho} \sqrt{\frac{a_{1} a_{2}}{\sigma_{1} \sigma_{2}}}\right) \exp \left(-n \frac{\frac{a_{1}}{\frac{\sigma_{1}}{1}+\frac{a_{2}}{\sigma_{2}}}}{1-\rho}\right)
\end{aligned}
$$




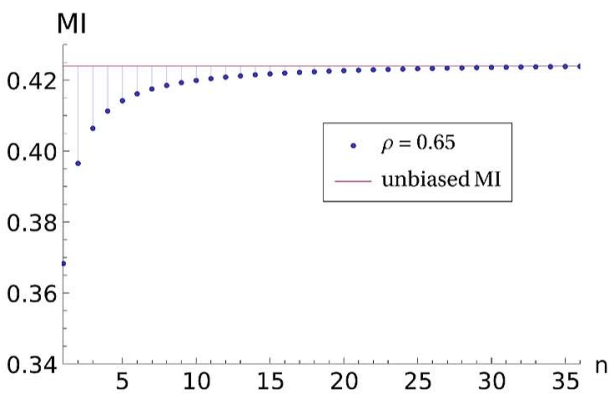

(a)

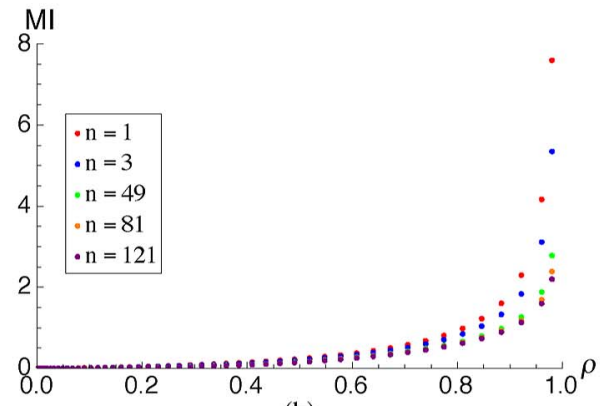

(b)

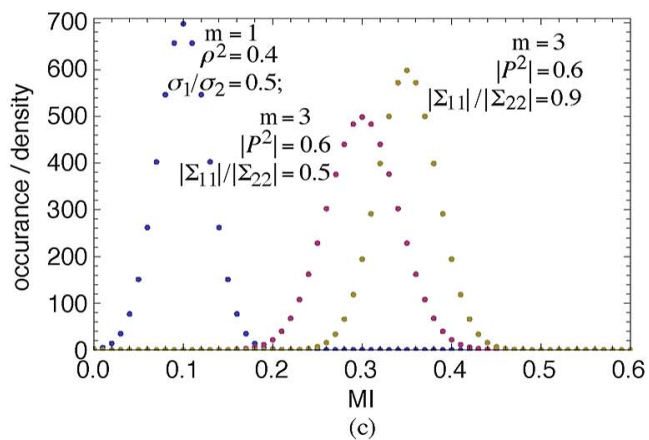

(c)

Fig. 2. (a) MI between temporal single-channel $(m=1)$ SAR images simulated with the parameters of $\sigma_{1}=1, \sigma_{2}=1.5$, and $\rho=0.65$ for different levels of $n$. (b) MI between temporal single-channel SAR images as a function of different levels of normalized correlation coefficients with different levels of $n$. (c) Simulated MI pdfs for different numbers of channels and levels of correlation with $n=9$.

$E\left\{A_{i i}\right\}=\Sigma_{i i}$, with $i=1,2$, the following decision statistics can be obtained:

$$
\begin{gathered}
\mathcal{D}_{\mathrm{MI}}^{n}\left(A_{11} ; A_{22}\right)=-\operatorname{tr}\left(\frac{2 n P^{2}}{I_{m}-P^{2}}\right)-n \log \left(\left|I_{m}-P^{2}\right|\right) \\
+E\left\{\log \left({ }_{0} \tilde{F}_{1}(n, C)\right)\right\} .
\end{gathered}
$$

$E\left\{\log \left({ }_{0} \tilde{F}_{1}(n, C)\right)\right\}$ can easily be obtained from (10) by spatial averaging. At this point, it can be seen from (17) that the decision statistic $\mathcal{D}_{\mathrm{MI}}^{n}\left(A_{11} ; A_{22}\right)$ is a function of the true correlation matrix $P^{2}$, which means that the estimated correlation matrix $R^{2}$ and $\mathcal{D}_{\mathrm{MI}}^{n}\left(A_{11} ; A_{22}\right)$ quantify the coherent similarity between temporal acquisitions by partly sharing the same original information. As expected, after substituting $m=1$ and (10) into (17), (17) converges to the MI of the bivariate Gamma distribution [24].

\section{A. Estimation Performance}

This section evaluates the MI estimation based on different numbers of samples and levels of correlation based on simulations. The number of Monte Carlo runs is 1000 for all figures presented in this section. The first simulation [Fig. 2(a)] shows the unbiased estimate of the MI related to the number of estimation samples $n$. From this figure, it can be concluded that the MI is asymptotically unbiased for a large number of samples. The second simulation [Fig. 2(b)], plotting the MI versus the correlation, shows the interest of the MI for low correlated temporal polarimetric pairs, which have much less variance than the correlated pairs, particularly for less number of samples. These simulation results, which are in line with [27], show that reliable estimation of the MI can be obtained for values of $n$ larger than $5 \times 5$. Fig. 2(c) shows a comparison of the simulated MI pdfs for different numbers of channels.

$$
\begin{aligned}
\mathcal{D}_{\mathrm{MI}}^{n}\left(A_{11} ; A_{22}\right)= & \int_{A_{11}, A_{22} \geq 0} \log \left(\frac{\operatorname{etr}\left(-n\left(\Sigma_{11 \mid 22}^{-1} A_{11}+\Sigma_{22 \mid 11}^{-1} A_{22}-\Sigma_{11}^{-1} A_{11}-\Sigma_{22}^{-1} A_{22}\right)\right)}{\left|I_{m}-P^{2}\right|^{n}}{ }_{0} \tilde{F}_{1}(n, C)\right) p\left(A_{11}, A_{22}\right) d \vec{A} \\
= & \int \log \left(\operatorname{etr}\left(-n\left(\frac{\Sigma_{11}^{-1} A_{11} P^{2}}{I_{m}-P^{2}}+\frac{\Sigma_{22}^{-1} A_{22} P^{2}}{I_{m}-P^{2}}\right)\right)\right) p\left(A_{11}, A_{22}\right) d \vec{A} \\
& +\int \log \left({ }_{0} \tilde{F}_{1}(n, C)\right) p\left(A_{11}, A_{22}\right) d \vec{A}-\int \log \left(\left|I_{m}-P^{2}\right|^{n}\right) p\left(A_{11}, A_{22}\right) d \vec{A} \\
= & -\operatorname{tr}\left(E\left\{A_{11}\right\} n \frac{\Sigma_{11}^{-1} P^{2}}{I_{m}-P^{2}}\right)-\operatorname{tr}\left(E\left\{A_{22}\right\} n \frac{\Sigma_{22}^{-1} P^{2}}{I_{m}-P^{2}}\right)+E\left\{\log \left({ }_{0} \tilde{F}_{1}(n, C)\right)\right\}-n \log \left(\left|I_{m}-P^{2}\right|\right)
\end{aligned}
$$




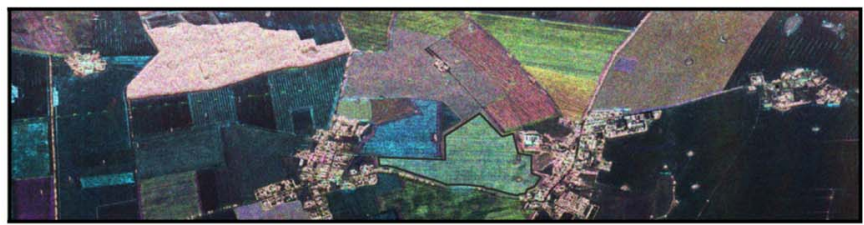

(a)

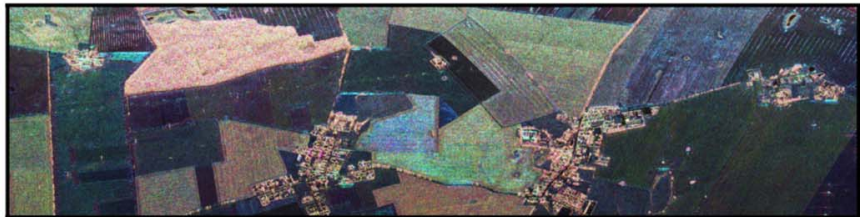

(b)

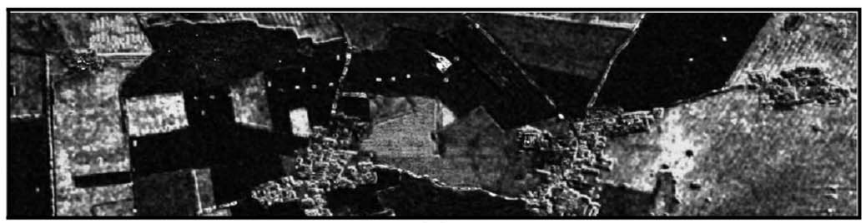

(c)

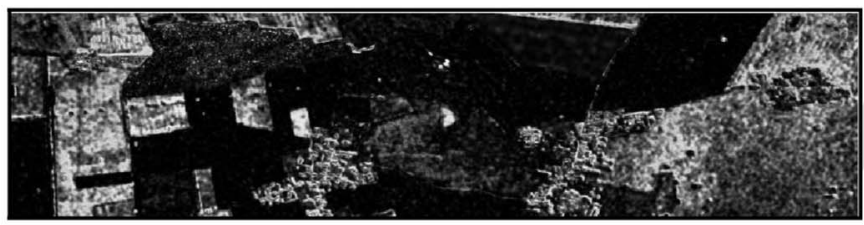

(d)

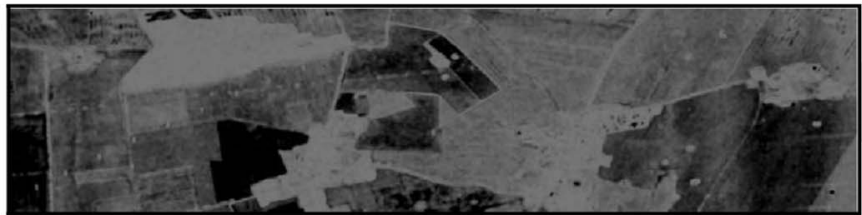

(e)

Fig. 3. PolSAR image color composition (red-HV, green-HH, blue-VV) of AgriSAR scenes captured (a) on April 19 and (b) on August 2. The black area shows the location of a maize field which is used in Section IV-C for ROC analysis. MI between April and August (c) PolInSAR and (d) InSAR images with $H H$-polarized channels. (e) MLRT of the PolInSAR image calculated based on (20). The results range from dark to bright (no change) depending on the amount of change. The MI values vary between 0 and 34 for the PolInSAR data set and between 0 and 28 for the InSAR data set, whereas the MLRT $(-\log Q)$ values vary between 0 and 7 .

As expected, by increasing the correlation and the number of channels, the mean of the MI increases. A higher generalized variance ratio $\left|\Sigma_{11}\right| /\left|\Sigma_{22}\right|$ shifts the pdfs to the right and, in parallel, reduces the MI variance (i.e., more deterministic scatterers).

\section{EXPERIMENTAL RESUlTS}

The proposed coherent similarity measure (17) is applied to fully polarimetric images acquired by the airborne E-SAR system of the German Aerospace Center (DLR) in the frame of the AgriSAR project [28]. PolInSAR data sets with 30- and 0 -m spatial baseline configurations were collected over the Görmin test site, located in northern Germany, during a whole vegetation growth period, between two acquisition dates on April 19 [Fig. 3(a)] and August 2 [Fig. 3(b)]. The sequence of acquisitions used in this work is listed in Table I. The last data set is used to investigate the influence of volumetric effect
TABLE I

E-SAR L-BAND ACQUISITIONS OVER THE GÖRMIN Agricultural Test Site. Range PiXel Spacing $\sim 1.5 \mathrm{~m}$ AZIMUTH PIXEL SPACING $\sim 3 \mathrm{~m}$

\begin{tabular}{|c|l|c|}
\hline & Temporal pairs & Baseline \\
\hline I & $19^{t h}$ April - $2^{\text {nd }}$ August & $0 \mathrm{~m}$ \\
\hline II & $11^{t h}$ May - $16^{t h}$ May & $0 \mathrm{~m}$ \\
\hline III & $11^{t h}$ May - $24^{t h}$ May & $0 \mathrm{~m}$ \\
\hline IV & $11^{t h}$ May - $21^{t h}$ June & $0 \mathrm{~m}$ \\
\hline V & $11^{t h}$ May - $26^{t h}$ July & $0 \mathrm{~m}$ \\
\hline VI & $19^{t h}$ April - $19^{t h}$ April & $30 \mathrm{~m}$ \\
\hline
\end{tabular}

on the coherent similarity, whereas the other ones are used to monitor temporal stability.

Experimental results with real data include three parts. In the first part, the proposed coherent similarity measure is applied into the entire test site using PolInSAR and InSAR data sets as reported in Fig. 3(c) and (d), respectively. In the second part, the significance of the features of the new coherent similarity parameter is highlighted using histograms over selected areas involved. This part evaluates the proposed measure through different combinations of polarimetric channels over three different agricultural fields. In the third part, the performance of the proposed measure for temporal scene characterization is evaluated by comparison with the well-known change detector called the MLRT.

\section{A. Global Analysis of the MI}

Section III-A and the experimental results using real and simulated data indicate that the estimation accuracy of polarimetric covariance matrices increases with the number of estimation samples. However, as discussed in the literature, the use of larger window sizes with fixed shapes may give unsatisfactory results, since the stationary/homogeneous assumption is often no longer valid and the accuracy of final estimates can decrease. Because of this, different covariance matrix estimation methods can be considered [29], [30]. Here, a region growing technique explained in [30] has been used instead of a fixed window size. In this method, only six intensity values from PolInSAR data are used to decide on the number of samples for maximumlikelihood covariance estimation. After an initial three-sample complex multilooking, the adaptive growing technique has been applied in order to supply homogeneity conditions for the estimation of the sample covariance matrices $A_{11}$ and $A_{22}$. Then, the unknown asymptotic parameters $\Sigma_{11}, \Sigma_{22}$, and $P^{2}$, which parameterize the pdf $p\left(A_{11}, A_{22}\right)$, can be estimated by maximum-likelihood rule as explained in the Appendix. In practice, the estimation of nonrandom variables may be relaxed: The random variables $A_{11}$ and $A_{22}$ are estimated by an initial nine-sample multilooking; then, the expectation variables can be calculated from the covariance matrices obtained by the method [30]. Here, it is worth noting that the estimated matrices by the practical technique are essentially the same as the matrices obtained directly employing the maximum-likelihood rule explained in the Appendix. Even though the maximumlikelihood estimation (MLE) of nonrandom parameters is given in the Appendix, the way to estimate the nonrandom variables is let to the reader. 


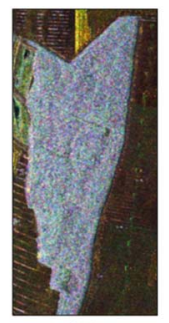

(a)

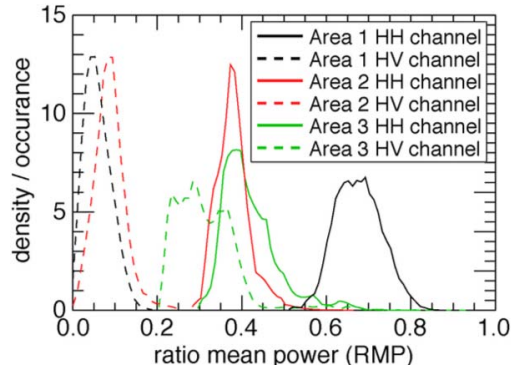

(d)

(b)
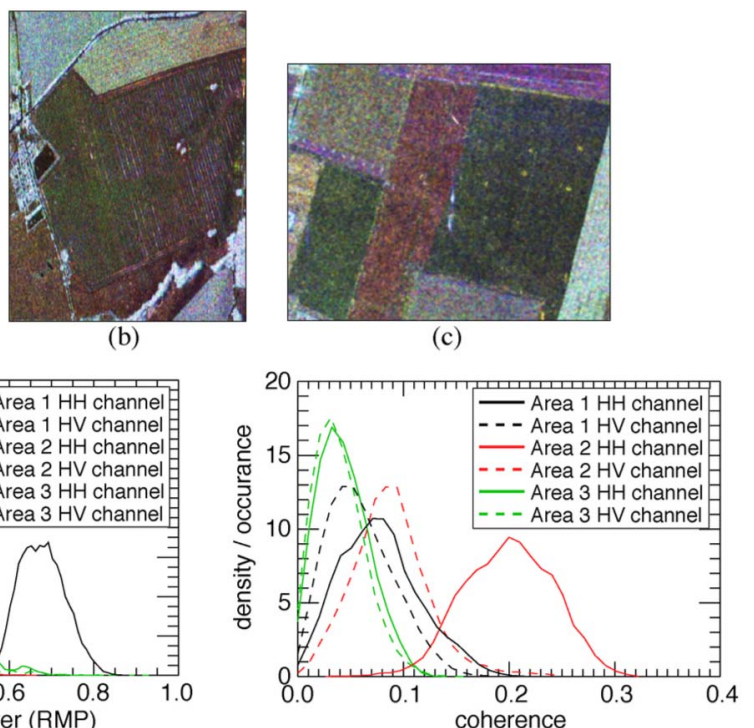

(e)

Fig. 4. Examples of agricultural fields illustrating the study areas by RGB polarimetric color composition (red-VV, green-HV, blue - HH) and the histograms of the intensity ratio and the coherence images with $H H$ and $H V$ polarizations. (a) Area 1. (b) Area 2. (c) Area 3. (d) Histogram of the intensity ratio. (e) Histogram of coherences.

The proposed coherent similarity measure is applied to PolInSAR and InSAR (with $H H$-polarization channels) images having 135-day temporal resolution to scale temporal change scenario, and they are shown in Fig. 3(c) and (d), respectively. The results range from dark to bright (no change) depending on the amount of temporal scene change. From these figures, one may easily observe that PolInSAR data supply a more detailed geometrical structure than the one with the InSAR image, and both measures have large values for residential areas, showing no significant difference between the fully polarimetric and single-channel images. The two most apparent differences between the results are that the full PolInSAR coherent similarity measure appears darker at identifying the large change in the fields having a volumetric decorrelation while the InSAR data set appears relatively lighter at identifying the small change and that the field of periodic surfaces (sometimes Bragg scattering over periodic surfaces) shows more delimited structures having high MI via the PolInSAR image than via the InSAR image. The first difference is easily explained by low degrees of correlation over some agricultural fields (e.g., forest) due to the high levels of volume decorrelation in PolInSAR acquisition. Since the physical parameters like roughness and geometric features can be extracted better with coherent techniques, the second difference appears.

\section{B. Analysis of the MI Through Selected Agricultural Fields}

In order to focus on the role of the MI in change detection applications, three fields with different crop types, which are shown in Fig. 4(a)-(c) by polarimetric red-green-blue (RGB) color composition, have been selected based on their differences in harvest/sowing time, crop volume, and structure. Fig. 4(d) and (e) shows the histograms of the intensity ratio and the coherence of these selected areas from data set I. The intensity ratio, known as the generalized variance ratio in terms of statistics, has been demonstrated the ability to distinguish different crops by identifying changes in the mean backscatter power [31]. The coherence has also been used in change detection applications such as urban monitoring and the effect of floods [32], [33]. As seen from these histograms, there are many types of temporal scene change, and each type can have a different effect on change detection analysis. Defining the type of change to be detected is as important as choosing the change detector. Considering these, the primary interest, through the three selected agricultural fields, is to figure out the behavior of MI as a temporal scene change detector, which allows the input of single and fully polarimetric images and multiple polarizations in different representations. The MI is applied using single polarizations $(H H, H V$, and $V V)$ and combinations of two polarizations $V V-H V$ known as partial PolInSAR data and fully polarimetric data. For single-polarized images, the MI makes use of the bivariate Gamma distribution (13). However, for PolInSAR and partial PolInSAR images, the MI uses the derived pdf (12).

The temporal evolution of the data set for each multidimensional channel combination is obtained with respect to the new coherent similarity measure, as expounded in Section III. Fig. 5 shows the histograms of $\mathcal{D}_{\mathrm{MI}}^{n}\left(A_{11} ; A_{22}\right)$ extracted from different channel configurations over the selected three agricultural fields. Several effects can be observed. First, not surprisingly, the observed MI values depend on the used polarization combinations. Second, $\mathcal{D}_{\mathrm{MI}}^{n}\left(A_{11} ; A_{22}\right)$ increases with high coherence. A difference in the range of the MI values depending on the crop type of polarimetric scattering mechanism is seen. The presence of correlations between temporal $\mathrm{HH}$ and temporal $\mathrm{VV}$ channels over area 2 affects the histogram of $\mathcal{D}_{\mathrm{MI}}^{n}$ of area 2 which is easily distinguished from those of area 1 and area 3. Although the mean values of the MI for area 2 in Fig. 5(a) with $m=2$ and in Fig. 5(c) with $m=3$ are very close, the variance decrease by increasing number of channels is seen. This result is confirmed in Fig. 2(c), which shows the decrease of the MI variance with increasing number of channels. 


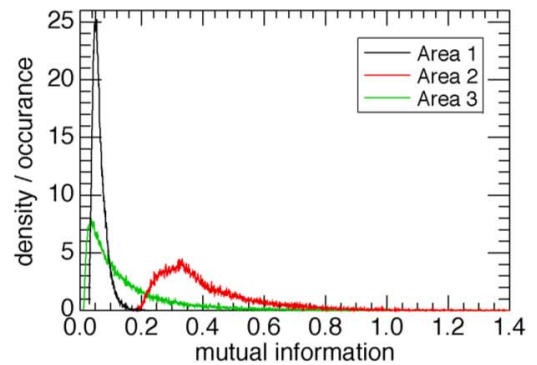

(a)

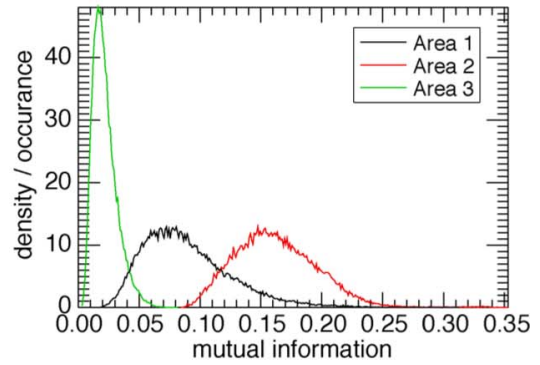

(b)

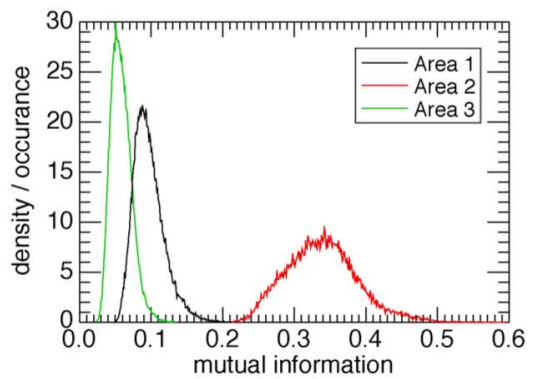

(c)

Fig. 5. Reconstructed probability distribution (histogram) of the decision statistic $\mathcal{D}^{n}\left(A_{11} ; A_{22}\right)$ associated to the selected areas in Fig. 4 through different channel configurations, i.e., (a) partial PolInSAR with $H H-V V$ channels, (b) partial PollnSAR with $H H-H V$ channels, and (c) PolInSAR. It can be stated that there is no any partial PolSAR imaging mode including $H H-V V$-polarized channels. This combination is shown here to highlight that the change detector varies based on the scattering mechanism (polarimetric properties) of the scene.

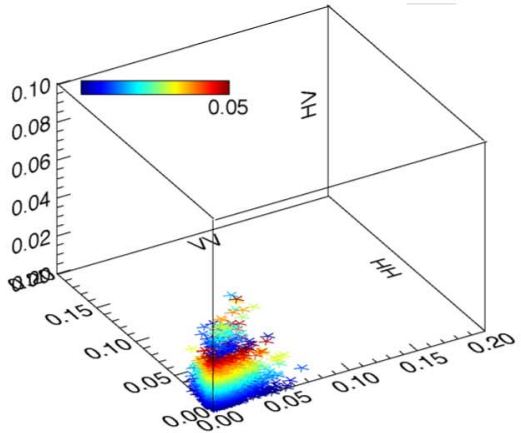

(a)

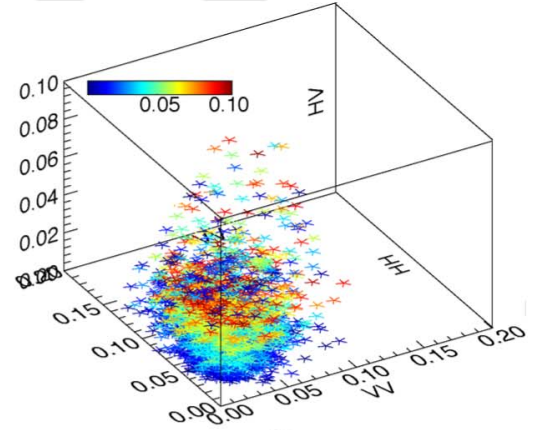

(b)

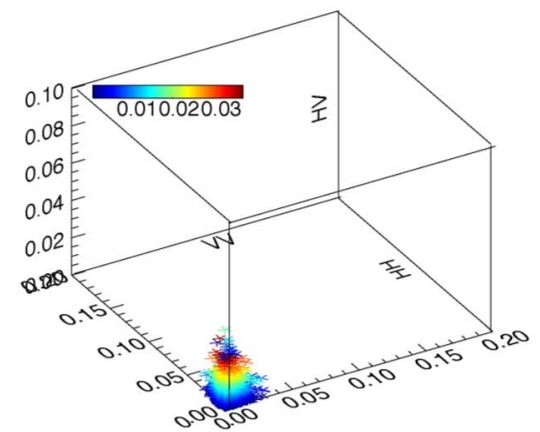

(c)

Fig. 6. Three-dimensional scatterplots showing an association of decision statistic $\mathcal{D}_{\mathrm{MI}}^{n}\left(a_{1} ; a_{2}\right)$ between $H H, H V$, and $V V$ temporal InSAR images through the three selected areas in Fig. 4. The color bar of each plot is scaled based on $\mathcal{D}_{\mathrm{MI}}^{n}\left(a_{1} ; a_{2}\right)$ obtained by InSAR mode with $H V$ polarization. (a) Area 1. (b) Area 2. (c) Area 3.

The 3-D scatterplots in Fig. 6 allow one to see the association of $\mathcal{D}_{\mathrm{MI}}^{n}\left(a_{1} ; a_{2}\right)$ values between InSAR images with $H H, V V$, and $H V$ polarizations which are separately plotted on each axis. Considering all three selected areas, $\mathcal{D}_{\mathrm{MI}}^{n}$ obtained from temporal InSAR with $H V$ polarization is lower than those of $H H$ and $V V$ polarizations. $\mathcal{D}_{\mathrm{MI}}^{n}\left(a_{1} ; a_{2}\right)$ through area 1 and area 3 shows a small coincidence in their spatial extent and location. However, $\mathcal{D}_{\mathrm{MI}}^{n}\left(a_{1} ; a_{2}\right)$ through area 2 spreads over a wide range of 3-D space, and $\mathcal{D}_{\mathrm{MI}}^{n}$ is greater in area 2 than in area 1 and area 3 for all polarizations. The comparison of the plots in Fig. 6 makes it clear that, in the cases of area 3 and area 1 , polarimetry does not have a strong influence on the 


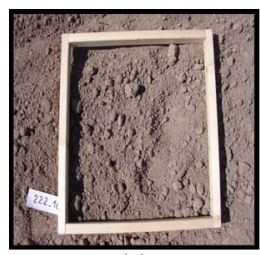

(a)

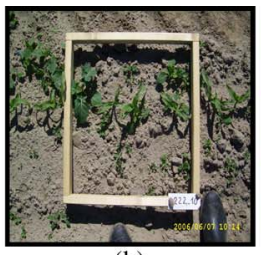

(b)

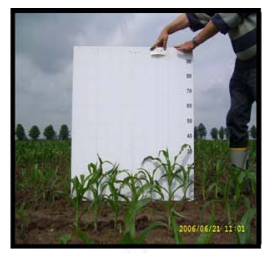

(c)

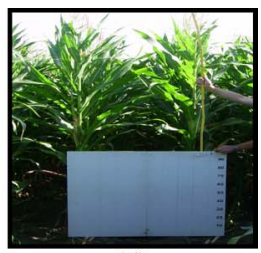

(d)

Fig. 7. Temporal photographs of the maize field which were taken simultaneously with PolSAR acquisitions in Table I. (a) April 16, 2006. (b) May 24, 2006. (c) June 21, 2006. (d) July 26, 2006.

temporal variation analysis. However, for the case of area 2, the different combinations of polarimetric channels result in different ranges of the MI values.

This set of plots illustrates a number of important issues. First and of great significance, even though the new coherent similarity measure is a simple function of the cross-correlation parameter, known as coherence, it does not take only cross-correlation parameters into account. It is the MI between two complex covariance matrices following the Wishart distribution whose random variables are their diagonal terms (mean backscatterer powers) and the real and imaginary parts of the upper offdiagonal terms (cross-correlation parameters). Second, it can be applied to any type of data such as polarimetric, interferometric, multifrequency, or any combination of these. As expected from multidimensional analysis, MI decreases with decreasing dimensionality if the whole channels have the same temporal characteristics. More precisely, the MI between two temporal images should be smaller if one only considers InSAR data instead of PolInSAR data if there is no difference between temporal behaviors of polarimetric channels. Third, the MI variation through selected areas might be simply explained by the coherence and the intensity ratio maps from a statistical point of view. However, in the presence of detailed information about vegetation geometry (e.g., orientation and dimension of branches) and soil roughness, this issue can also be clarified from a physical point of view.

\section{Gain of the Change Detection Application}

In terms of testing dependence of two sets of variables, any algorithm, as the one just proposed, depends only on the matrices of the canonical correlation $R^{2}$ and the variance ratio $A_{11} A_{22}^{-1}$, which are, in the remote sensing field, known as the interferometric coherence matrix and the mean power ratio, respectively. For example, as detailed in [17, Ch. 8], the wellknown likelihood ratio test about investigating the equality of temporal polarimetric covariance matrices is

$$
\Delta=\left(\frac{|A|}{\left|A_{11}\right|\left|A_{22}\right|}\right)^{n} \text {. }
$$

Writing the equality of $|A|=\left|A_{11 \mid 22}\left\|A_{22 \mid 11}\right\| I_{m}-R^{2}\right|$ and $R^{2}=A_{11}^{-1} A_{12} A_{22}^{-1} A_{21}$ into (18) implies that

$$
\begin{aligned}
\Delta & =\left|I_{m}-A_{11}^{-1} A_{12} A_{22}^{-1} A_{21}\right|^{n} \\
& =\prod_{i=1}^{m}\left(1-r_{i}^{2}\right)^{n}
\end{aligned}
$$

where $m$ is the dimension of the acquisition at time $t_{i}$ and $0 \leq r_{1}^{2}<r_{2}^{2}<\cdots<r_{m}^{2}<1$ are the nonzero eigenvalues of $R^{2}$.
Additionally, the polarimetric change detector of the MLRT as a function of $A_{11} A_{22}^{-1}$ is [8], [9]

$$
Q=\frac{\left|A_{11}\right|^{n}\left|A_{22}\right|^{n}}{\left|A_{11}+A_{22}\right|^{2 n}}=\frac{\left|A_{11} A_{22}^{-1}\right|^{n}}{\left|I_{m}+A_{11} A_{22}^{-1}\right|^{2 n}} .
$$

Thus, the final goal of this study is to highlight the advantage of using the MI compared to (20)—which is shown in Fig. 3(e) - as a change detector. To evaluate the performance of the proposed detector derived in (17) and (20), the metric of detection algorithm performance called receiver operating characteristic (ROC) curves can be used. ROC curves are the curves of probability of detection (PD) versus probability of false alarm (PFA) and describe the detection performance by expressing the tradeoff between PD and PFA. For practically analyzing the changed (PD) and unchanged (PFA) hypotheses, the relatively flat maize agricultural field indicated by the black line in Fig. 3(a) is chosen. For formulating the unchanged scene hypothesis (PFA) related to the bare field, the acquisition vector $\vec{k}=\left[\vec{k}_{1} \vec{k}_{2}\right]$ is formed by PolSAR images acquired on May 11 and 16. For the changed hypothesis related to the presence of vegetation, the PolInSAR data sets III, IV, and V in Table I were used. Observing the photographs of the maize field in Fig. 7, which were simultaneously taken with PolSAR acquisitions, it can be noticed that the maize field was sown in the beginning of May and starts to grow in the end of July. It is interesting to underline the fact that there is no significant mean power change for this field until the end of July. However, there is a significant change in temporal scattering mechanisms. Fig. 8 shows the ROC plots of (17) and (20) obtained by applying appropriate thresholds. It is clear that (17) has a significantly better detection performance for short temporal analysis, allowing the scene temporal changes to be more readily discerned. However, on the time of harvesting, the MLRT change detector has also a very good detection performance because the fully developed maize field has produced significant changes in the pixel intensities. The ROC curve example can be increased by choosing other agricultural fields. In that case, it can be seen that the PFA is low also for the MLRT technique where the type of change is easily detected by computing the mean value. However, when a more complex type of change occurs, the proposed measure has a lower PFA than the one by the MLRT. This could be because, compared to the MLRT, the MI takes also into account the interferometric information, making the proposed technique also attractive for defining man-made scene disturbances.

\section{Discussions AND CONCLUSION}

In this paper, a new coherent similarity measure based on the second-order statistics of the acquisition vector has been 


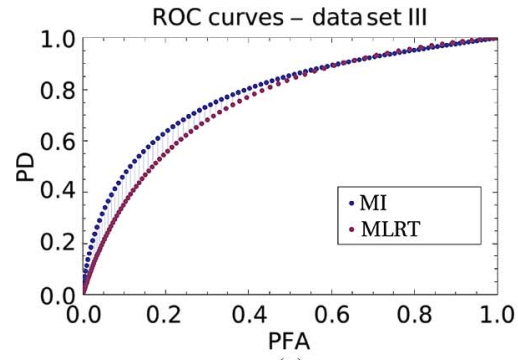

(a)

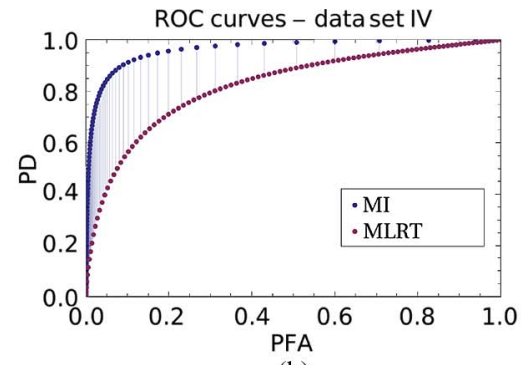

(b)

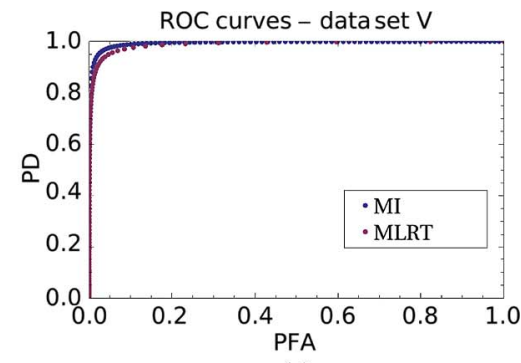

(c)

Fig. 8. ROC curves of (17) and (20) by means of change detection. It can be noted that, since the ROC curves are a function of the thresholds, to make a fair comparison, the threshold values are chosen by the same rule. The thresholds applied in each case range to achieve a fixed number of false alarms in unchanged samples. (a) May 24, 2006. (b) June 21, 2006. (c) July 26, 2006.

proposed for temporal multichannel SAR images, particularly for a PolInSAR image. This new parameter makes use of the MI between Wishart processes over time. For InSAR images, the proposed parameter agrees with the one provided in [24], and it is the MI of the bivariate Gamma distribution. Although, in this paper, the MI has analytically been derived for PolInSAR images, since the mathematical formulation is general, it can be implemented into some kind of multivariate remote sensing image such as multispectral optical and interferometric images. Moreover, a new simple joint distribution function of two polarimetric covariance matrices, so-called complex Wishart matrices, has been derived based on temporal SAR acquisition vector history. As corollaries to this new result, explicit expressions for the statistics of the joint distribution of temporal Wishart processes can be interesting for future temporal applications as well. Capitalizing on the foregoing distribution, it becomes possible the removal of the bias in temporal multichannel parameters.

The aim of this work is to provide a unique scalar parameter that allows one to represent and scale the coherent similarity occurring between the acquisitions at time 1 and time 2 and to utilize this scalar parameter as a tool for change detection application. Since change detection application-hence the implementation of the proposed metric - is application dependent, it is avoided to give a specific example of application such as identifying areas inundated by flood, discriminating crop types, and urban and glacier monitoring. Although defining assumptions and constraints for an application is left to the readers, the new coherent similarity measure has been implemented to fully polarimetric data to characterize temporal scene change. The analysis carried out with real data provided interesting results for each different polarimetric channel combination and confirmed the capabilities of the proposed technique for temporal information characterization. The implementation of the proposed detector into different polarimetric channel combinations allows studying the spatial and temporal behavior of the data through different polarizations. This analysis showed that the development of an optimal approach for the exploitation of the temporal change may be application dependent. To put in a nutshell, only single-channel acquisition with any polarization is enough to characterize temporal behavior, while it is necessary to work on polarimetric data to characterize temporal behavior at other times.

The proposed detector has also been compared to the wellknown and powerful change detector known as the MLRT and has been shown to have a more detailed change detection analysis than this classical algorithm based on real data. As a concrete example, if the aim is to detect military tracks in a forest, the MLRT has a very small false-alarm rate based on a big change in mean backscatter power. However, to detect a much more complicated temporal scene scenario, the proposed measure can better characterize the temporal change. The strength of MI is in its ability to find similarity between not only temporal polarimetric information-as the MLRT does-but also interferometric information.

Further research is needed to figure out the influence of implementation issues, such as multiscale optimization, the number of samples, and cumulant-based methods. Furthermore, the robustness of the method with respect to speckle has to be investigated.

\section{APPENDIX MLE}

Let $X=\left(X^{1}, X^{2}, \ldots, X^{q}\right)$ denote $q$ independent vectors representing $q$ observations $X^{i}=\operatorname{vec}\left(A_{11}^{i}, A_{22}^{i}\right)$ following the distribution of $p\left(A_{11}, A_{22}\right)$, and it is interesting to estimate an unknown nonrandom parameter $\theta$ including $\Sigma_{11}$, $\Sigma_{22}$, and $P^{2}$ which parameterize the pdf $p\left(A_{11}, A_{22}\right)$. Then, $p_{\theta}\left(X^{1}, X^{2}, \ldots, X^{q}\right)$ is a function of $\theta$ alone, and the value of $\theta$ that maximizes the aforementioned pdf $\left(p_{\theta}\left(X^{1}, X^{2}, \ldots, X^{q}\right)\right)$ is the most likely values for vector $\theta$. Under the assumption that $X$ includes independent and identically distributed random variables, the maximum log-likelihood function can be written using $p_{\theta}\left(X^{1}, X^{2}, \ldots, X^{q}\right)$ with the help of (12) as

$$
\begin{aligned}
& \mathcal{L}(X ; \theta) \\
&=\sum_{i=1}^{q} \log p_{\theta}\left(X^{i}\right) \\
&=\sum_{i=1}^{q}\left(\log \left[\operatorname{etr}\left(-n \frac{\Sigma_{22}^{-1} A_{22}^{i}+\Sigma_{11}^{-1} A_{11}^{i}}{I-P^{2}}\right)\right]\right. \\
&+\log \left[\frac{n^{2 m n}\left|A_{11}^{i} A_{22}^{i}\right|^{n-m}}{\left|\Sigma_{11} \Sigma_{22}\right|^{n}\left|I-P^{2}\right|^{n} 22 \tilde{\Gamma}_{m}(n) \tilde{\Gamma}_{m}(n)}\right] \\
&+\log \left[{ } _ { 0 } \tilde { F } _ { 1 } \left(n, n^{2} A_{11}^{i}{ }^{1 / 2} \Sigma_{11 \mid 22}^{-1}{ }^{-1}{ }_{12} \Sigma_{22}^{-1} A_{22}^{i}\right.\right.
\end{aligned}
$$


Substituting $\Sigma_{11 \mid 22}=\Sigma_{11}\left(I_{m}-P^{2}\right), P^{2}=\Sigma_{11}^{-1} \Sigma_{12} \Sigma_{22}^{-1} \Sigma_{21}$, and the means

$$
\bar{A}_{11}=\frac{1}{q} \sum_{i=1}^{q} A_{11}^{i} \quad \bar{A}_{22}=\frac{1}{q} \sum_{i=1}^{q} A_{22}^{i}
$$

into (21) and eliminating the constants give the following loglikelihood function:

$$
\begin{aligned}
\mathcal{L}(X ; \theta)= & \sum_{i=1}^{q} \log _{0} \tilde{F}_{1}(\underbrace{n, \underbrace{2} \frac{P^{2} \Sigma_{11}^{-1} A_{11}^{i} \Sigma_{22}^{-1} A_{22}^{i}}{\left(I-P^{2}\right)\left(I-P^{2}\right)}}_{M}) \\
& -n q\left(\frac{\Sigma_{22}^{-1} \bar{A}_{22}}{I-P^{2}}+\frac{\Sigma_{11}^{-1} \bar{A}_{11}}{I-P^{2}}+\log \left(\left|\Sigma_{11} \Sigma_{22}\right|\left|I-P^{2}\right|\right)\right) .
\end{aligned}
$$

Differentiating with respect to the parameter vector $\theta$ yields

$$
\begin{aligned}
& \frac{d \mathcal{L}(X ; \theta)}{d \Sigma_{11}} \\
& =n q \frac{\Sigma_{11}^{-1} \bar{A}_{11} \Sigma_{11}^{-1}}{I_{m}-P^{2}}-n q \Sigma_{11}^{-1} \\
& -\frac{n^{2} \Sigma_{11}^{-1} P^{2}}{\left(I_{m}-P^{2}\right)^{2}}\left(\sum_{i=1}^{q} \Sigma_{22}^{-1} A_{22}^{i} \Sigma_{11}^{-1} A_{11}^{i} \frac{{ }_{0} \tilde{F}_{1}(n+1, M)}{{ }_{0} \tilde{F}_{1}(n, M)}\right) \\
& =n q \frac{\bar{A}_{11}}{I_{m}-P^{2}}-n q \Sigma_{11} \\
& -\frac{n^{2} \Sigma_{11} P^{2}}{\left(I_{m}-P^{2}\right)^{2}}\left(\sum_{i=1}^{q} \Sigma_{22}^{-1} A_{22}^{i} \Sigma_{11}^{-1} A_{11}^{i} \frac{{ }_{0} \tilde{F}_{1}(n+1, M)}{{ }_{0} \tilde{F}_{1}(n, M)}\right)=0 \\
& \frac{d \mathcal{L}(X ; \theta)}{d \Sigma_{22}} \\
& =n q \frac{\Sigma_{22}^{-1} \bar{A}_{22} \Sigma_{22}^{-1}}{I_{m}-P^{2}}-n q \Sigma_{22}^{-1} \\
& -\frac{n^{2} \Sigma_{22}^{-1} P^{2}}{\left(I_{m}-P^{2}\right)^{2}}\left(\sum_{i=1}^{q} \Sigma_{22}^{-1} A_{22}^{i} \Sigma_{11}^{-1} A_{11}^{i} \frac{{ }_{0} \tilde{F}_{1}(n+1, M)}{{ }_{0} \tilde{F}_{1}(n, M)}\right) \\
& =n q \frac{\bar{A}_{22}}{I_{m}-P^{2}}-n q \Sigma_{22} \\
& -\frac{n^{2} \Sigma_{22} P^{2}}{\left(I_{m}-P^{2}\right)^{2}}\left(\sum_{i=1}^{q} \Sigma_{22}^{-1} A_{22}^{i} \Sigma_{11}^{-1} A_{11}^{i} \frac{{ }_{0} \tilde{F}_{1}(n+1, M)}{{ }_{0} \tilde{F}_{1}(n, M)}\right)=0 \\
& \frac{d \mathcal{L}(X ; \theta)}{d P^{2}} \\
& =n q \frac{\Sigma_{11}^{-1} \bar{A}_{11}}{\left(I-P^{2}\right)^{2}}+n q \frac{\Sigma_{22}^{-1} \bar{A}_{22}}{\left(I-P^{2}\right)^{2}}-\frac{n q}{n^{2}\left(I_{m}-P^{2}\right)} \\
& -\frac{\left(I_{m}+P^{2}\right)}{\left(I-P^{2}\right)^{3}}\left(\sum_{i=1}^{q} \Sigma_{22}^{-1} A_{22}^{i} \Sigma_{11}^{-1} A_{11}^{i} \frac{{ }_{0} \tilde{F}_{1}(n+1, M)}{{ }_{0} \tilde{F}_{1}(n, M)}\right) \\
& =\frac{n q \Sigma_{11}^{-1} \bar{A}_{11}}{\left(I-P^{2}\right)}+\frac{n q \Sigma_{22}^{-1} \bar{A}_{22}}{\left(I-P^{2}\right)} \\
& -n^{2} \frac{I_{m}+P^{2}}{I_{m}-P^{2}}\left(\sum_{i=1}^{q} \Sigma_{22}^{-1} A_{22}^{i} \Sigma_{11}^{-1} A_{11}^{i} \frac{{ }_{0} \tilde{F}_{1}(n+1, M)}{{ }_{0} \tilde{F}_{1}(n, M)}\right) \\
& -n q I_{m}=0 \text {. }
\end{aligned}
$$

To perform the derivations in (23), the following rules of the differentials of matrices were used [34]:

$$
\begin{aligned}
& \frac{d}{d t}|A|=|A| A^{-1} \\
& \frac{d}{d t}\left[A^{-1}\right]=-A^{-1}\left(\frac{d}{d t}[A]\right) A^{-1} \\
& d(A+B)=d A+d B \text { the rule of linearity } \\
& \frac{d}{d x}\left({ }_{p} F_{q}\left(a_{1}, \ldots, a_{p} ; c_{1}, \ldots, c_{q} ; x\right)\right) \\
& \quad=\frac{\prod_{k=1}^{p} a_{k}}{\prod_{j=1}^{q} c_{j}} F_{q}\left(a_{1}+1, \ldots, a_{p}+1 ; c_{1}+1, \ldots, c_{q}+1 ; x\right) .
\end{aligned}
$$

Solving the equation of $d \mathcal{L}(X ; \theta) / \Sigma_{11}$ for $\Sigma_{11}$ in terms of $\Sigma_{22}$, substituting this expression for $\Sigma_{11}$ into the equation of $d \mathcal{L}(X ; \theta) / \Sigma_{22}$, and simplifying the given expression give the desired MLE of $\Sigma_{11}$ and $\Sigma_{22}$ as

$$
\begin{array}{ll}
\underbrace{\widehat{\Sigma}_{11}=\bar{A}_{11}}_{\text {MLE }} & A_{11}=\frac{1}{q} \sum_{i=1}^{q} A_{11}^{i} \\
\underbrace{\widehat{\Sigma}_{22}=\bar{A}_{22}}_{\text {MLE }} & A_{22}=\frac{1}{q} \sum_{i=1}^{q} A_{22}^{i} .
\end{array}
$$

Substituting the MLE of $\Sigma_{11}$ and $\Sigma_{22}$ into $d \mathcal{L}(X ; \theta) / d P^{2}$, the MLE function of the $P^{2}$ matrix corresponding to temporal correlation is obtained

$$
\underbrace{\widehat{P}^{2}=I_{m}-P^{2}-\frac{n}{q}\left(\sum_{i=1}^{q} \bar{A}_{22}^{-1} A_{22}^{i} \bar{A}_{11}^{-1} A_{11}^{i} \frac{\left.{ }_{0} \frac{\tilde{F}_{1}(n+1, \bar{M})}{{ }_{0} \tilde{F}_{1}(n, \bar{M})}\right)}{{ }(n)}\right.}_{\text {MLE }}
$$

where

$$
\bar{M}=\frac{n^{2} P^{2} \bar{A}_{11}^{-1} A_{11}^{i} \bar{A}_{22}^{-1} A_{22}^{i}}{\left(I_{m}-P^{2}\right)\left(I_{m}-P^{2}\right)} .
$$

The root matrix of (25) is the MLE of $P^{2}$ with the condition of $0<P^{2}<I_{m}$. The desired MLE of $P^{2}$ can be calculated by the iterative method Newton fractal which is a boundary set in the complex plane that is characterized by the Newton-Raphson method applied to a fixed polynomial $p(P) \in$ $\mathcal{C}[P]$. Newton's fractal method can often converge remarkably quickly, particularly if the iteration begins "sufficiently near" the desired root. In this case, it is efficient to start to iteration with $R^{2}=A_{11}^{-1 / 2} A_{12} A_{22}^{-1} A_{21} A_{11}^{-1 / 2}$ calculated from $2 m \times 2 m$ sample matrix $A$.

\section{ACKNOWLEDGMENT}

The authors would like to thank the anonymous reviewers for the constructive discussions and suggestions to improve the quality of this paper. 


\section{REFERENCES}

[1] R. Dianat and S. Kasaei, "Change detection in optical remote sensing images using difference-based methods and spatial information," IEEE Geosci. Remote Sens. Lett., vol. 7, no. 1, pp. 215-219, Jan. 2010.

[2] F. Bovolo, L. Bruzzone, and M. Marconcini, "A novel approach to unsupervised change detection based on a semi-supervised SVM and a similarity measure," IEEE Trans. Geosci. Remote Sens., vol. 46, no. 7, pp. 2070-2082, Jun. 2008.

[3] G. Margarit, J. J. Mallorquí, and L. Pipia, "Polarimetric characterization and temporal stability analysis of urban target scattering," IEEE Trans. Geosci. Remote Sens., vol. 48, no. 4, pp. 2038-2048, Apr. 2010.

[4] D. Kim, W. M. Moon, and Y. S. Kim, "Application of TerraSAR-X data for emergent oil-spill monitoring," IEEE Trans. Geosci. Remote Sens., vol. 48, no. 2, pp. 852-863, Feb. 2010.

[5] L. Ferro-Famil, A. Reigber, E. Pottier, and W. M. Börner, "Scene characterization using subaperture polarimetric SAR data," IEEE Trans. Geosci. Remote Sens., vol. 41, no. 10, pp. 2264-2276, Oct. 2003.

[6] E. Erten, A. Reigber, and O. Hellwich, "Generation of three-dimensional deformation maps from InSAR data using spectral diversity techniques," ISPRS J. Photogramm. Remote Sens., vol. 65, no. 4, pp. 388-394, Jul. 2010.

[7] P. R. Kersten, J. S. Lee, and T. L. Ainsworth, "A comparison of change detection statistics in POLSAR images," in Proc. IGARSS, Seoul, Korea, 2005, vol. 7, pp. 4836-4839.

[8] K. Conradsen, A. A. Nielsen, J. Schou, and H. Skriver, "A test statistic in the complex Wishart distribution and its application to change detection in polarimetric SAR data," IEEE Trans. Geosci. Remote Sens., vol. 41, no. 1, pp. 4-19, Feb. 2003.

[9] L. Novak, "Change detection for multi-polarization, multi-pass SAR," in Proc. SPIE Conf. Algorithms Synthetic Aperture Radar Imagery, 2005, pp. 26-30.

[10] J. Morio, P. Réfrégier, F. Goudail, P. C. Dubois-Fernandez, and X. Dupuis, "Information theory-based approach for contrast analysis in polarimetric and/or interferometric SAR images," IEEE Trans. Geosci. Remote Sens., vol. 46, no. 8, pp. 2185-2196, Jul. 2008.

[11] J. Inglada, G. Mercier, and T. Cnes, "A new statistical similarity measure for change detection in multitemporal SAR images and its extension to multiscale change analysis," IEEE Trans. Geosci. Remote Sens., vol. 45, pt. 2, no. 5, pp. 1432-1445, Apr. 2007.

[12] F. Maes, A. Collignon, D. Vandermeulen, G. Marchal, and P. Suetens, "Multimodality image registration by maximization of mutual information," IEEE Trans. Med. Imag., vol. 16, no. 2, pp. 187-198, Apr. 1997.

[13] P. Reinartz, R. Muller, P. Schwind, S. Suri, and R. Bamler, "Orthorectification of VHR optical satellite data exploiting the geometric accuracy of TerraSAR-X data," ISPRS J. Photogramm. Remote Sens., vol. 66, no. 1, pp. 124-132, Jan. 2011.

[14] S. Suri and P. Reinartz, "Mutual-information-based registration of TerraSAR-X and Ikonos imagery in urban areas," IEEE Trans. Geosci. Remote Sens., vol. 48, no. 2, pp. 939-949, Feb. 2010.

[15] R. J. Radke, S. Andra, O. Al-Kofahi, and B. Roysam, "Image change detection algorithms: A systematic survey," IEEE Trans. Image Process., vol. 14, no. 3, pp. 294-307, Feb. 2005.

[16] J. Lee and E. Pottier, Polarimetric Radar Imaging: From Basics to Applications. Boca Raton, FL: CRC Press, 2009.

[17] R. J. Muirhead, Aspects of Multivariate Statistic Theory. Hoboken, NJ: Wiley, 1982.

[18] L. Ferro-Famil, E. Pottier, and J. S. Lee, "Unsupervised classification of multifrequency and fullypolarimetric SAR images based on the H/A/Alpha-Wishart classifier," IEEE Trans. Geosci. Remote Sens., vol. 39, no. 11, pp. 2332-2342, Nov. 2001.

[19] M. McKay and I. Collings, "Statistical properties of complex noncentral Wishart matrices and MIMO capacity," in Proc. ISIT, 2005, pp. 785-789.

[20] P. J. Smith and L. M. Garth, "Distribution and characteristic functions for correlated complex Wishart matrices," J. Multivariate Anal., vol. 98, no. 4, pp. 661-677, Apr. 2007.

[21] G. Iliopoulos, "UMVU estimation of the ratio of powers of normal generalized variances under correlation," J. Multivariate Anal., vol. 99, no. 6, pp. 1051-1069, Jul. 2008.

[22] E. Erten, "Information theory of multi-temporal SAR systems with application to motion detection and change detection," Ph.D. dissertation, DLR, Wessling, Germany, 2010, DLR-FB 2010-38.

[23] H. Holm and M. Alouini, "Sum and difference of two squared correlated Nakagami variates in connection with the McKay distribution," IEEE Trans. Commun., vol. 52, no. 8, pp. 1367-1376, Jul. 2004.

[24] F. Chatelain, J. Y. Tourneret, J. Inglada, and A. Ferrari, "Bivariate gamma distributions for image registration and change detection," IEEE Trans. Image Process., vol. 16, no. 7, pp. 1796-1806, Jul. 2007.
[25] Mathematica 8 homepage. [Online]. Available: http://reference.wolfram. $\mathrm{com} / \mathrm{mathematica/ref/KolmogorovSmirnovTest.html}$

[26] A. Papoulis, Probability, Random Variables, and Stochastic Processes, 4th ed. Singapore: McGraw-Hill, 2002.

[27] R. Moddemeijer, "A statistic to estimate the variance of the histogrambased mutual information estimator based on dependent pairs of observations," Signal Process., vol. 75, no. 1, pp. 51-63, Jan. 1999.

[28] I. Hajnsek, T. Jagdhuber, H. Schon, and K. P. Papathanassiou, "Potential of estimating soil moisture under vegetation cover by means of PolSAR," IEEE Trans. Geosci. Remote Sens., vol. 47, no. 2, pp. 442-454, Feb. 2009.

[29] J. S. Lee, S. R. Cloude, K. P. Papathanassiou, M. R. Grunes, and I. H. Woodhouse, "Speckle filtering and coherence estimation of polarimetric SAR interferometry data for forest applications," IEEE Trans. Geosci. Remote Sens., vol. 41, no. 10, pp. 2254-2263, Oct. 2003.

[30] G. Vasile, E. Trouvé, J. Lee, and V. Buzuloiu, "Intensity-driven adaptiveneighborhood technique for polarimetric and interferometric SAR parameters estimation," IEEE Trans. Geosci. Remote Sens., vol. 44, no. 6, pp. 1609-1621, Jun. 2006.

[31] E. J. M. Rignot and J. J. Van Zyl, "Change detection techniques for ERS-1 SAR data," IEEE Trans. Geosci. Remote Sens., vol. 31, no. 4, pp. 896906, Aug. 1993.

[32] D. Corr, "Coherent change detection for urban development monitoring," in Proc. IEE Colloq. Radar Interferometry, 2002, pp. 6/1-6/6.

[33] D. Geudtner, R. Winter, and P. W. Vachon, "Flood monitoring using ERS-1 SAR interferometry coherence maps," in Proc. IGARSS-Remote Sensing for a Sustainable Future, 2002, vol. 2, pp. 966-968.

[34] I. S. Gradshteyn and I. M. Ryzhik, Table of Integrals, Series, and Products. New York: Academic, 2007.

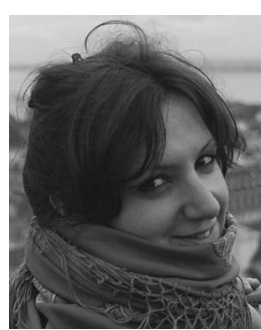

Esra Erten received the B.S. degree in geodesy and photogrammetry engineering and the M.S.E.E. degree in satellite communication and remote sensing from Istanbul Technical University, Istanbul, Turkey, in 2003 and 2005, respectively, and the Ph.D. degree from the Technische Universität Berlin, Berlin, Germany, in 2010.

From April 2008 to June 2010, she was with the High-Frequency Institute, German Aerospace Center (DLR), Wessling, Germany, where she worked on information theory for multichannel synthetic aperture radar (SAR) images. Since 2011, she has been with the Chair of Earth Observation and Remote Sensing, Institute of Environmental Engineering, Swiss Federal Institute of Technology (ETH) Zurich, Zurich, Switzerland. Her research interests include information extraction and image understanding from SAR and optical images, particularly information theory, multivariate statistics, polarimetry, interferometry, and hyperspectral imaging.

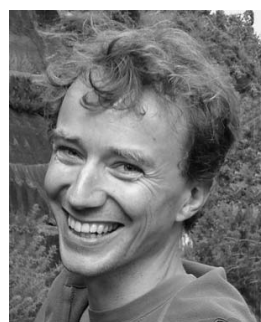

Andreas Reigber (M'02-SM'10) was born in München, Germany, in 1970 . He received the Diploma degree in physics from the University of Konstanz, Konstanz, Germany, in 1997, the Ph.D. degree from the University of Stuttgart, Stuttgart, Germany, in 2001, and the Habilitation from the Technische Universität Berlin (TUB), Berlin, Germany, in 2008.

From 1996 to 2000, he was with the Microwaves and Radar Institute, German Aerospace Center (DLR), Wessling, Germany, working in the field of polarimetric synthetic aperture radar (SAR) tomography. In 2001, he joined the Antenna, Radar and Telecommunication Laboratory, University of Rennes 1, Rennes, France, for postdoctoral research on radar polarimetry and polarimetric interferometry. From 2002 to 2007, he was a Research Associate with the Department of Computer Vision and Remote Sensing, TUB. Since 2008, he has been with the Microwaves and Radar Institute, DLR, where he is currently the Head of the SAR Technology Department and directing the airborne SAR activities of the institute. His current main research interests include the various aspects of multimodal SAR, like SAR interferometry, SAR polarimetry, SAR tomography, and time-frequency analysis, as well as filtering and classification aspects of high-resolution SAR data.

Dr. Reigber was the recipient of the European Conference on Synthetic Aperture Radar 2000 Student Prize Paper Award for an article on SAR remote sensing of forests, the IEEE GEOSCIENCE AND REMOTE SENSING TRANSACTIONS (GRSS) Prize Paper Award in 2001 for a work on polarimetric SAR tomography, and the IEEE GRSS Letters Prize Paper Award in 2006 for a work on multipass SAR processing. 


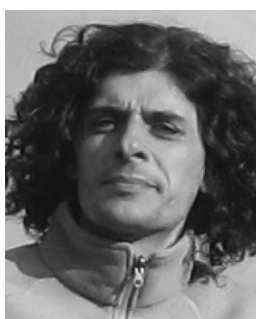

Laurent Ferro-Famil (M'00) received the Laurea degree in electronics systems and computer engineering, the M.S. degree in electronics, and the $\mathrm{Ph} . \mathrm{D}$. degree from the University of Nantes, Nantes, France, in 1996, 1996, and 2000, respectively.

In 2001, he became an Associate Professor with the University of Rennes 1, Rennes, France, where he has been a Full Professor since 2011 and is currently the Head of the Radar Polarimetry Remote Sensing Group, within the Institute of Electronics and Telecommunications of Rennes. His current activities in education are concerned with analog electronics, digital communications, microwave theory, signal processing, and polarimetric synthetic aperture radar (SAR) remote sensing. He is particularly interested in polarimetric SAR signal processing, radar polarimetry theory, and natural media remote sensing using multibaseline polarimetric-interferometric SAR data, with application to classification, electromagnetic scattering modeling and physical parameter retrieval, time-frequency analysis, and 3-D reconstruction of environments.

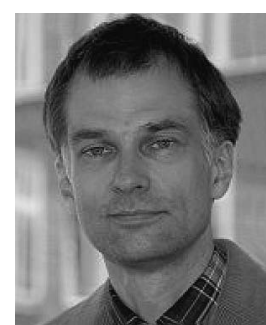

Olaf Hellwich (M'98-SM'06) was born in 1962. He received the B.S. degree in surveying engineering from the University of New Brunswick, Fredericton, $\mathrm{NB}$, and the Ph.D. degree from the Technische Universität München, München, Germany, in 1997.

He headed the Remote Sensing Group, Department of Photogrammetry and Remote Sensing, Technische Universität München. Since 2001, he has been a Professor with the Technische Universität Berlin (TUB), Berlin, Germany, initially for photogrammetry and cartography and since 2004 for computer vision and remote sensing. He is currently the Dean of the Faculty of Electrical Engineering and Computer Science, TUB. His research interests are in 3-D object reconstruction, e.g., from trinocular video sequences, object recognition, e.g., real-time head pose estimation, and synthetic aperture radar remote sensing, e.g., for surface motion estimation.

Dr. Hellwich was the recipient of the Hansa Luftbild Prize of the German Society for Photogrammetry and Remote Sensing in 2000. 